\title{
Descripción del cromosoma profásico en la meiosis I de Bostryx conspersus
}

\section{Description of the profasic chromosome in Meiosis I of Bostryx conspersus}

\author{
María Siles-Vallejos ${ }^{1}$, Olga Bracamonte G. ${ }^{1}$, Alberto López S. ${ }^{1}$, Betty Shiga ${ }^{2}$ y \\ Misael Guevara P. ${ }^{1}$
}

1Laboratorio de Citogenética Facultad de Ciencias Biológicas, Universidad Nacions Blogicas, Sniversidad Nacional Mayor de San Marcos. Apartado 110058 Lima 11, Perú.

2 Laboratorio de Fisiología Animal, Facultad de Ciencias Biológicas, UNMSM

Email María Siles:

msilesv@unmsm.edu.pe,

Email Alberto López:

alopezs@unmsm.edu.pe

Presentado: $\quad 12 / 02 / 2010$

Aceptado: $\quad$ 23/07/2010

Publicado online: $25 / 08 / 2011$

\section{Resumen}

Bostryx es uno de los géneros de mayor diversidad dentro de los Orthalicidae (Gastropoda), siendo B. conspersus una especie endémica del ecosistema de Lomas, del desierto Costero peruano. En el presente trabajo los cromosomas de la profase I de meiosis de los espermatocitos de Bostryx conspersus son descritos.

Palabras clave: Desierto costero del Perú; ecosistema de Lomas costeras, cromosoma; profase, meiosis I.

\section{Abstract}

Bostryx is among the most diverse genera within the Orthalicidae (Gastropoda). Bostryx conspersus is endemic of the Lomas ecosystem in Peruvian Coastal Desert. In this paper, chromosomes in prophase I of meiosis of spermatocytes Bostryx conspersus are described.

Keywords: Peru's coastal desert, coastal ecosystem Lomas, chromosome; prophase, meiosis I.

\section{Introducción}

En el ecosistema costero de Lomas del Perú han sido reportadas diferentes especies endémicas, entre las que destacan algunos caracoles; sobre estos caracoles se han realizado estudios anatómicos (Lopez 1998), taxonómicos, ecológicos y de distribución geográfica (Ramírez, 1988); también existen estudios sobre la diversidad genética a nivel molecular (Ramírez 2004; Ramírez et al. 2009), pero no se han reportado trabajos sobre los cromosomas de estos moluscos.

Bostryx es uno de los géneros de mayor diversidad entre los Orthalicidae, siendo $B$. conspersus una de las especie endémica típica de la malacofauna de Lomas en el Perú (Ramírez 1988).

Los estudios citogenéticos pueden ser de gran utilidad en el discernimiento de poblaciones, e indispensables en la identificación de razas cariotípicas, ya que las alteraciones en el número y en la estructura cromosómica del cariotipo durante el curso de la evolución desempeñan una función importante en el proceso del aislamiento reproductivo y por lo tanto, de la especiación. Por lo tanto conocer las características del cromosoma profásico durante la primera meiosis es importante para deducir la presencia y formación de gametos viables en la especie.

En el presente trabajo describimos los cromosomas profásicos de la primera meiosis de los espermatocitos de Bostryx conspersus, lo que nos va a permitir inferir el comportamiento del cromosoma durante la meiosis, y establecer el número haploide de cromosomas.

\section{Material y métodos}

Los ejemplares Bostryx conspersus (Sowerby, 1833) fueron colectados ad libitum entre mayo y octubre de 2007 en dos localidades del complejo de las Lomas de Atocongo, ubicadas en la Provincia de Lima: Lomas de El Lúcumo (12¹2'23.8”S, $\left.76^{\circ} 53^{\prime} 43^{\prime \prime W}\right)$ y Paraíso (1208'20.2”S, 76 55'23.4”W).

Los especimenes adultos, con abertura de la boca mayor de $15 \mathrm{~mm}$ fueron inyectados con $0,5 \mathrm{~mL}$ de colchicina $0,2 \%$ por 24 horas; posteriormente se procedió a su relajamiento sumergiéndolos en una solución de nicotina por 12 horas. Se les extrajo la masa visceral y se colocó en solución fisiológica $(\mathrm{NaCl}$ 0,6\%). La disección se realizó bajo un estereoscopio, procediendo a separar la glándula hermafrodítica, la que fue hipotonizada en solución de $\mathrm{CINa}$ 0,2\%. Seguidamente se fijó el tejido gonadal en solución etanol-ácido acético (3:1) para transferirlos luego a solución Targa. Se disgregó el tejido, y se trasladó a láminas portaobjetos heladas agregándoles una gota de orceína lacto acética $2 \%$ por 15 minutos para luego realizar el aplastamiento.

La observación se realizó en un microscopio óptico, seleccionándose las mejores láminas para ser fotografiadas a 1000X con una cámara fotográfica digital de 7,2 megapixeles.

El número cromosómico fue determinado por conteo directo bajo el microscopio y en fotografías de núcleos en diacinesis. Se hicieron diagramas de las placas fotográficas seleccionadas.

Se seleccionaron las láminas para la identificación de cromosomas en profase I, teniendo en cuenta para la identificación de los mismos las configuraciones meióticas típicas.

\section{Resultados}

Se analizaron 34 láminas procedentes de 5 individuos (2 procedieron de Paraíso y 3 de El Lúcumo) contabilizándose 850 campos, encontrando que el número de bivalentes fluctúa entre 20 y 28. Se observaron células redondas, de diferente tamaño, identificadas como espermatogonias en interfase, el nucleolo no se coloreo con la orceína pero se pudo inferir su presencia (Fig. 1).

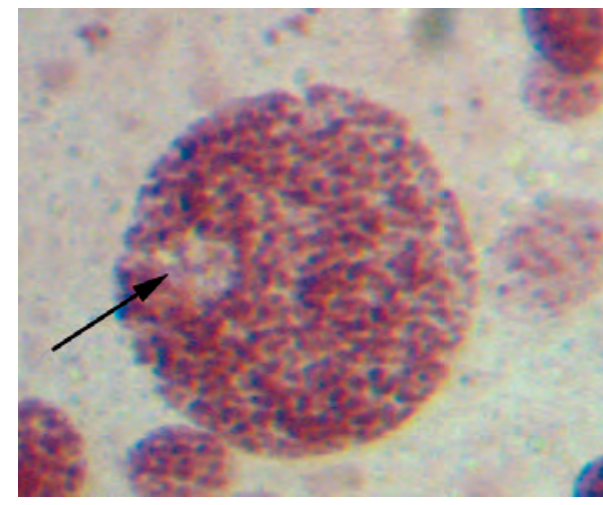

Figura 1.

Célula en interfase. La flecha indica el nucleolo que no se ha teñido con la orceina. Aumento 1000X. 


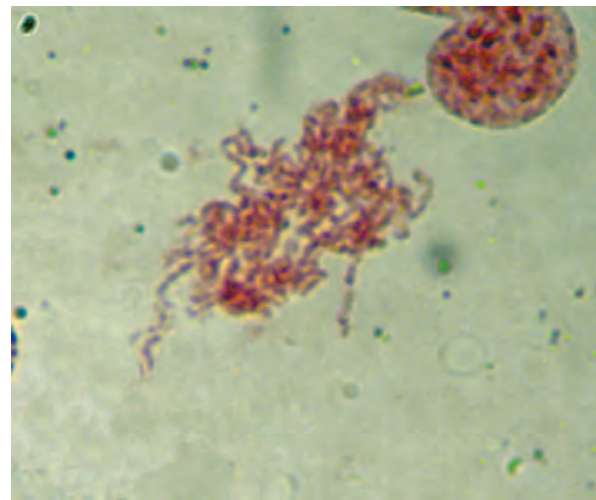

Figura 2.

Leptoteno. Obsérvese el enmarañado de la fibra, iniciando su condensación. Aumento $1000 \mathrm{X}$

En la primera profase meiótica no se han detectado irregularidades en los distintos estadios. Observamos leptotenos con la fibra de cromatina muy delgada, difusa y enmarañada (Fig. 2); cigotenos con la fibra un poco más gruesa y con bloques heterocromáticos grandes y muy teñidos (Fig. 3a). En los paquitenos tempranos se observan los bivalentes estrechamente apareados, luego se va haciendo evidente la presencia de las dos cromátidas por cromosoma (Fig. 3b), las siluetas de los bivalentes se observan irregulares y se evidencian unas estructuras intensamente teñidas que resaltan notoriamente en algún punto a lo largo de la fibra (Figs. 3 b y c). En algunos cromosomas se evidencia una pequeńa zona que no se tiñe, lo que podría corresponder al centrómero (Figs. 3b y c), las cuales no se observan claramente en todos los cromosomas probablemente debido a la condensación. En Los diplotenos se observan que los bivalentes empiezan a adoptar disposiciones en anillo o en cruz (Fig. 4).

El conteo de placas en diploteno y diacinesis, de individuos procedentes de ambas localidades, nos permite observar un

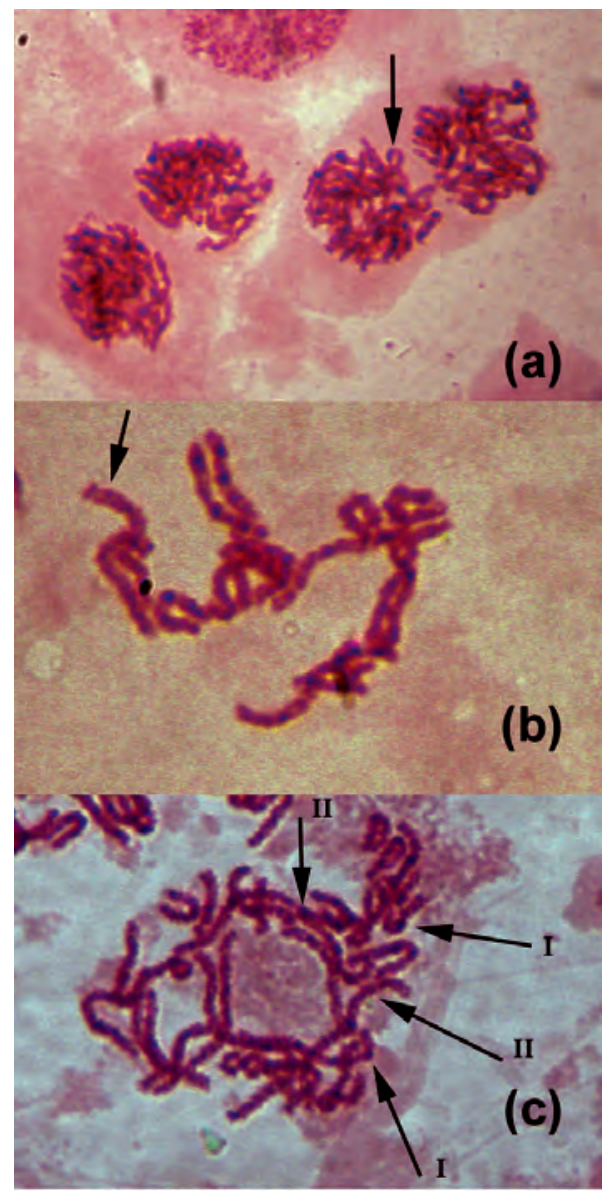

Figura 3.

(a) Cigotenos. La fibra se observa un poco más gruesa y empieza a notarse bloques heterocromáticos (flecha).

(b) Paquiteno. Se observan los homólogos apareados y la disposición de los cromómeros siguiendo un patrón (I).

(c) Paquiteno. Se observan los "knobs" (I) y las regiones centroméricas (II). Aumento 1000X.

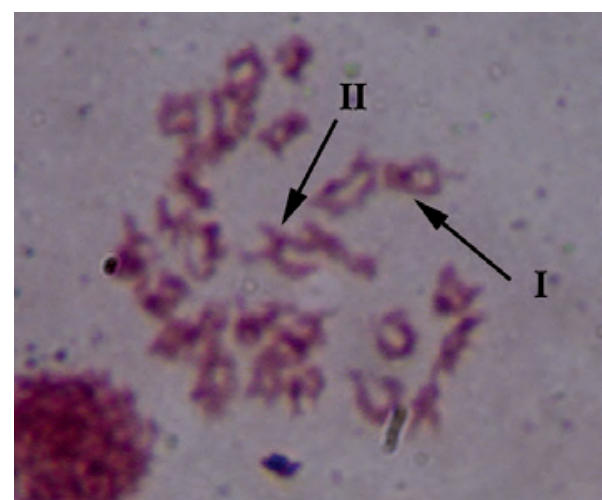

Figura 4.

Diploteno con bivalente en forma de anillo (I) y en cruz (II). Aumento 1000X.

Tabla 1. Número de cromosomas encontrado e 34 láminas en diploteno / diacinesis de Bostryx conspersus.

\begin{tabular}{lrrrrrrrr} 
Cromosomas & 21 & 22 & 23 & 24 & 25 & 26 & 27 & 28 \\
\cline { 2 - 8 } Frecuencia & 3 & 2 & 833 & 3 & 2 & 3 & 2 & 2 \\
\hline
\end{tabular}

número haploide de cromosomas que fluctuó entre 22 y 28, determinándose un número haploide de $\mathrm{n}=23$ (Tabla 1).La mayoría de los bivalentes presentaron forma de anillo, pero también se observaron elementos con la forma de barra o cruz. Se observaron quiasmas intersticiales y terminales (Fig. 5a y b). La mayoría de los cromosomas fueron de pequeño tamaño.

\section{Discusión}

Vitturi et al. (1982, 1988, 2002) mencionan que el número haploide de gasterópodos fluctúa entre 9 a 35 cromosomas y en pulmonados el número haploide fluctúa entre $\mathrm{n}=5$ y $\mathrm{n}=44$, siendo el promedio $n=24$.

En el presente trabajo el número haploide de B. conspersus seria $n=23$; en los casos en que se observó número diferente (placas con 19 ó 22 cromosomas y placas entre 24 a 28 cromosomas) podría deberse a la pérdida o rotura de bivalentes. Esto parece ser común entre los moluscos debido al pequeño tamaño de sus cromosomas y a la rapidez de su división, lo cual dificulta el estudio cromosómico (Viturri et al. 1988). Además, debemos precisar que esta dificultad se hace mayor por efecto de la técnica. En otros casos existía demasiada sobreposición entre bivalentes

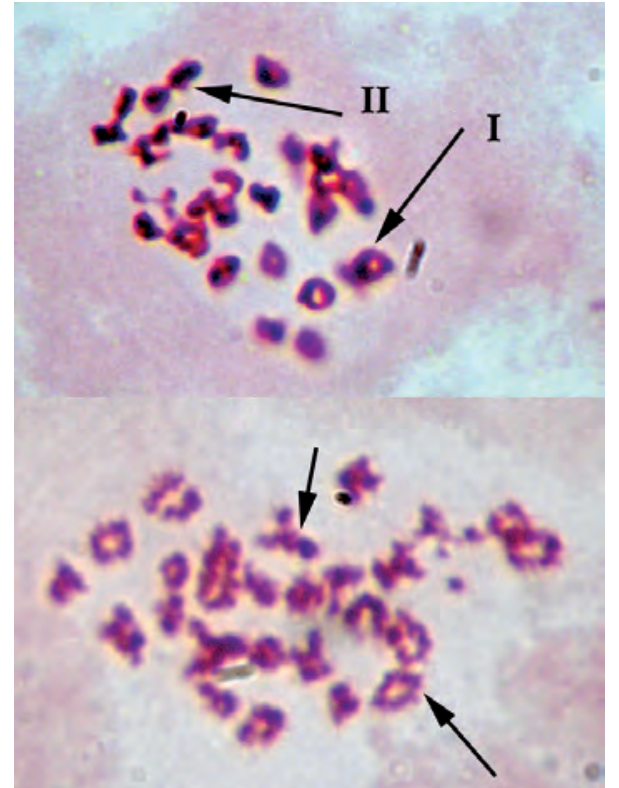

Figura 5.

(a) Diacinesis temprana, $n=23$ cromosomas bivalentes en anillo (I) y e barra (II). (b) Diacinesis tempraquiasmas terminales (I) y quiasmas intersticiales (II). Aumento 1000X. na. Se observan 
y en algunos casos, se observaban conjuntos cromosómicos de más de una placa meiótica. Esta desventaja ha sido observada en otros moluscos marinos, de agua dulce o terrestres (Vitturi et al. 1982, Vitturi et al. 1989, Chambers 1982).

Nuestro análisis sugiere las siguientes características citogenéticas: los leptotenos y cigotenos no muestran mayor diferencia, excepto el engrosamiento de la fibra. Esto ha sido observado en numerosas especies tanto en el reino animal como en el reino vegetal (Lacadena 1996). Los bivalentes del paquiteno temprano se observan largos e irregulares y conforme avanza el estadio se van acortando y engrosando, resaltando la silueta irregular. Esa irregularidad ha sido observada en otras especies de moluscos (Thiriot-Qiévreux 1990, Vitturi et al. 1982) concluyéndo que es común no sólo en moluscos sino en muchas especies animales y vegetales. De hecho, la silueta irregular se debe a la presencia de los cromómeros, que son engrosamientos de la fibra muy heteropicnóticos, y que permiten caracterizar a cada bivalente de acuerdo a su número y posición. Estos cromómeros se encuentran en los bivalentes paquiténicos de todas las especies vegetales y animales (Lacadena 1996). La estrecha relación de los bivalentes paquiténicos se debe a la sinapsis de los homólogos observada en la profase I, y que además se puede demostrar con claridad cuando se sigue el patrón de cromómeros en ambos homólogos. Esta conformación del bivalente paquiténico le da el aspecto de un cromosoma politénico de los núcleos interfásicos de las glándulas salivales de los dípteros.

En las láminas observadas no fue posible diferenciar cromatina sexual, la cual usualmente se hace evidente como un corpúsculo heteropicnótico adosado a la carioteca. Tampoco se han encontrado elementos heterotípicos como en la mayoría de los gasterópodos terrestres (hermafrodita) en los que no se observa la presencia de cromosomas sexuales (Viturri et al. 1982, Viturri \& Catalano 1989).

También en los bivalentes paquiténcos se observan regiones más heterocromáticas que posiblemente correspondan a los knobs o nudos, descritos en distintas especies vegetales y animales (Lacadena 1996) y considerados marcadores para identificar cromosomas y regiones específicas de éstos. B. conspersus tendría knobs muy heteropicnóticos aunque de pequeño tamaño, y en posición intermedia.
La mayoría de los bivalentes presentaron forma de anillo o cruz y otras pocas formas de barra, lo cual siguiere que la totalidad de los bivalentes fueron quiasmáticos siendo evidencia de recombinación. Se observaron bivalentes con quiasmas simples y con más de un quiasma, tanto terminales como intersticiales.

\section{Agradecimientos}

A Natalia Tong y Angie Uturunco por la disección de los especimenes. Al estudiante Carlos Díaz, por la colecta de los especimenes. A la Dra. Rina Ramírez por la identificación de la especie.

\section{Literatura Citada}

Chambers S. 1982. Chromosomal evidence of parallel evolution of shell sculpture pattern in Goniobasis. Evolution 16(1): 113-120.

Lacadena J.R. 1996. Citogenética. 1ed. Ed. Complutense S.A. España. $931 \mathrm{pp}$.

López F.; J. Pino. 1998. Análisis morfohistológico del sistema reproductor de Bostryx conspersus (Sowerby, 1833) (Gastropoda, Bulimidae) de las lomas de Pacta. Lima, Perú. Rev. peru. biol. 5(2): 148-143.

Ramírez, R. 1988. Morfología y Biología de Bostryx conspersus (SOWERBY) (MOLUSCA,BULIMULIDAE) en las lomas costeras del Perú Central. Rev. Bras. Zool. 5(4):609617.

Ramírez J.; R. Ramírez; P. Romero; A. Chumbe; P. Ramírez. 2009. Posición evolutiva de caracoles terrestres peruanos (Orthalicidae) entre los Stylommatophora (Mollusca: Gastropoda). Rev. peru. biol. 16(1): 051- 056.

Ramírez R. 2004. Sistemática e Filogeografia dos Moluscos do Ecossistema de "Lomas" do Deserto da Costa Central do Peru. Tese de Doutorado em Zoologia. Faculdade de Biociências, PUCRS, Porto Alegre, Brasil.

Thiriot-Quiévreux C. 1990. Karyotype analysis in several pelagic gastropods. Amer. Malac. Bull. 8(1):37-44.

Vitturi R.; Rasotto M., Farinella-Ferruzza N. 1982. The chromosomes of 16 molluscan species. Boll. Zol. 49: 61-71.

Vitturi R., E. Catalano, M. Macaluso y B. Zava. 1988. The karyotype of ittorina nitoides (Linnaeus, 1758) (Mollusca, Prosobranchia). Malacologia 29(2): 319 - 324.

Vitturi R., Colomba M., Castriota L., Belgrano A.M., Lannino A., Volpe N. 2002. Chromosome análisis using different staining techniques and fluorescent in situ hybridization in Cerithium vulgatum (Gastropoda: Cerithiidae) Hereditas 137:101-106 
